\title{
Solitary nodule of angiolymphoid hyperplasia with eosinophilia of the back masquerading as pyogenic granuloma
}

\author{
SHU-LIN LI ${ }^{1}$ and JIAN-DE HAN ${ }^{2}$ \\ ${ }^{1}$ Department of Dermatology, Shenzhen Zhongshan Urology Hospital, Shenzhen, Guangdong 518045; ${ }^{2}$ Department of \\ Dermatology, The First Affiliated Hospital of Sun Yat-sen University, Guangzhou, Guangdong 510080, P.R. China
}

Received May 17, 2017; Accepted September 1, 2017

DOI: $10.3892 / \mathrm{mco} .2017 .1423$

\begin{abstract}
Angiolymphoid hyperplasia with eosinophilia (ALHE) is a rare chronic inflammatory disorder of unknown etiology that most commonly presents as painless lymphadenopathy or subcutaneous masses in the head and neck region. The pathogenesis of the disease is not yet clear. The differential diagnosis is determined using characteristic histological features, such as significant vascular proliferation, lymphocytes and eosinophil inflammation in the dermis. Here is presented the case of a patient with a solitary nodule of ALHE masquerading as pyogenic granuloma (PG). A 47 year-old male patient was admitted with an isolated lump on the right side of the back, which had been present for $\sim 6$ months; the nodule appeared to have characteristics of PG and was $\sim 1.8 \times 1.0 \mathrm{~cm}^{2}$ in diameter. To the best of our knowledge, this is the first case report regarding ALHE arising in the back. The present report discusses the manifestation, histopathological features and treatment of the case.
\end{abstract}

\section{Introduction}

Angiolymphoid hyperplasia with eosinophilia (ALHE), also known as epithelioid hemangioma, is rare type of benign vascular hyperplastic lesion (1). This disease usually presents with pink-red/brown, dome-shaped, dermal papules or nodules on the head or neck, particularly near the ears and on the scalp (2). The lesion(s) may be associated with pain or pruritus. Uncommon symptoms include pulsation and spontaneous bleeding. It is unclear whether ALHE is a reactive or neoplastic disease. In its active phase, it can be misdiagnosed as an angiosarcoma; however, eosinophilia is not a usual feature of malignant angiosarcoma (3). Pyogenic granuloma (PG) is a small, eruptive, usually solitary, sessile or pedun-

Correspondence to: Dr Jian-De Han, Department of Dermatology, The First Affiliated Hospital of Sun Yat-sen University, 58 Zhongshan 2nd Road, Yuexiu, Guangzhou, Guangdong 510080, P.R. China E-mail: hanjd_gzb@21cn.net

Key words: eosinophilia, epithelioid hemangioma, granulomatous, pyogenic granuloma, Kimura's disease culated, friable papule. The lesion is common in children and occurs most often on an exposed surface. PG bleeds easily with the slightest trauma and, if removed superficially, may promptly recur. Recurring lesions may have one or numerous satellite lesions (2).

\section{Case report}

A 47-year-old East Asian male patient inadvertently noticed a protuberant, mildly pruritic, reddish colored nodule near the scapular region of the back present for the past 6 months. The patient was admitted to the Dermatology Department of Shenzhen Zhongshan Urological Hospital (Shenzhen, China) in November 2015. The patient gave history of accidental bleeding and blood scab formation from the nodule following trivial trauma such as rubbing of the skin. There was no history of fever, night sweats, weight loss or decreased appetite.

The general physical examination was unremarkable. Over the vicinage of scapular region was a round, reddish colored, protuberant nodule $\sim 1.8 \times 1 \mathrm{~cm}$ in size, which was firm in consistency with smooth overlying surface (Fig. 1). Surrounding the nodule, few prominent pilosebaceous follicles over a reddish brown base were observed. There was no axillary or inguinal lymphadenopathy and no hepatosplenomegaly. Routine blood investigations revealed Hb: $141 \mathrm{~g} / 1$, WBC: $6.3 \times 10^{9} / 1$, eosinophil count: $0.52 \times 10^{9} / 1$ (reference range, $0.02-0.52 \times 10^{9} / 1$ ), eosinophil count $\%: 8.3 \%(0.4-0.8 \%)$. The coagulation profile provided the following values: PT: $13.2 \mathrm{~S}, \mathrm{PT} \%$ : $98 \%$, APTT: $29 \mathrm{~S}$, TT: 15S, Fib2.71 g/l. Liver and renal function tests were normal.

Histopathological examination of the nodule revealed squamous epithelial hyperplasia, epithelial hyperplasia of the blood vessels, significant fibroplasia, and granulomatous tissue formation with a large number of eosinophils, plasmacytes and lymphocytes infiltrating the stroma (Fig. 2). CD34 was positive (Fig. 3) in proliferative blood vessels while the perivascular lymphocytes exhibited CD20 positivity (Fig. 4). The diagnosis of ALHE was determined on the basis of clinicopathological correlation.

\section{Discussion}

ALHE was first described by Wells and Whimster (4) in 1969, and the etiology and pathogenesis remains unclear. Certain scholars had considered benign tumor vascular tissue 


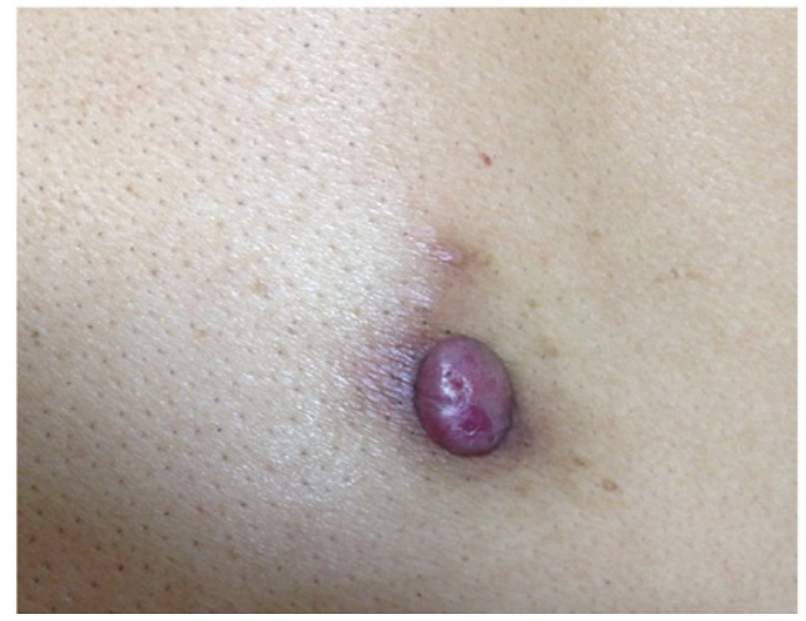

Figure 1. Angiolymphoid hyperplasia with eosinophilia atypically Exhibits a round, reddish, protuberant nodule $\sim 1.8 \times 1.0 \mathrm{~cm}^{2}$ in diameter, which was firm in consistency with a smooth overlying surface.

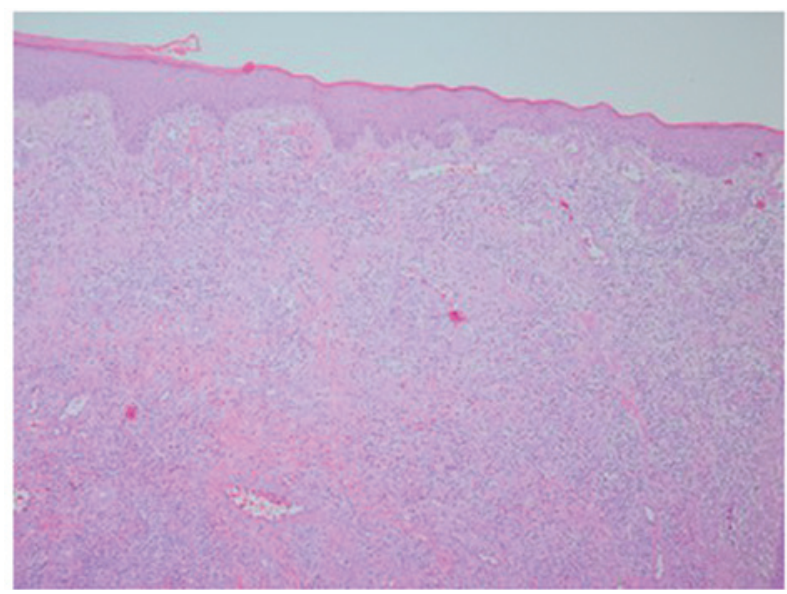

Figure 2. Squamous epithelial hyperplasia, blood vessels and epithelial fiber hyperplasia, hyperplasia of blood vessels of uneven size and thickness, branching blood vessels and the formation of granulation tissue. Inflammatory infiltrates included a large number of lymphocytes, eosinophils, plasmacytes and histocytes in the mesenchyme (hematoxylin and eosin; magnification, $\mathrm{x} 40$ ).

hyperplasia, or possible blood vessel reactive hyperplasia to external injury, infection (HTLV/HHV8), or symptoms of renin or high estrogen (5-7). The study observed that may be benign or malignant abnormal $\mathrm{T}$ lymphocyte proliferation $(8,9)$. ALHE and Kimura's disease were originally considered to be the same entity, but are now considered to be separate disease processes (3).

To date, ALHE cases have been reported worldwide, but the incidence is currently unknown. ALHE is uncommon but not rare, and presents most commonly in patients of Asian populations followed by Caucasians; ALHE may also be more common in Japan than in other countries. ALHE is more common in females; however, a male predominance has been noted in a prior Asian study (10). ALHE presents most commonly in patients aged $20-50$ years, with mean age of onset of 30-33 years. Overall, $~ 85 \%$ of lesions occur in the skin of the head and neck, with the majority on or near the ear, forehead or scalp (10). The typical clinical manifestations

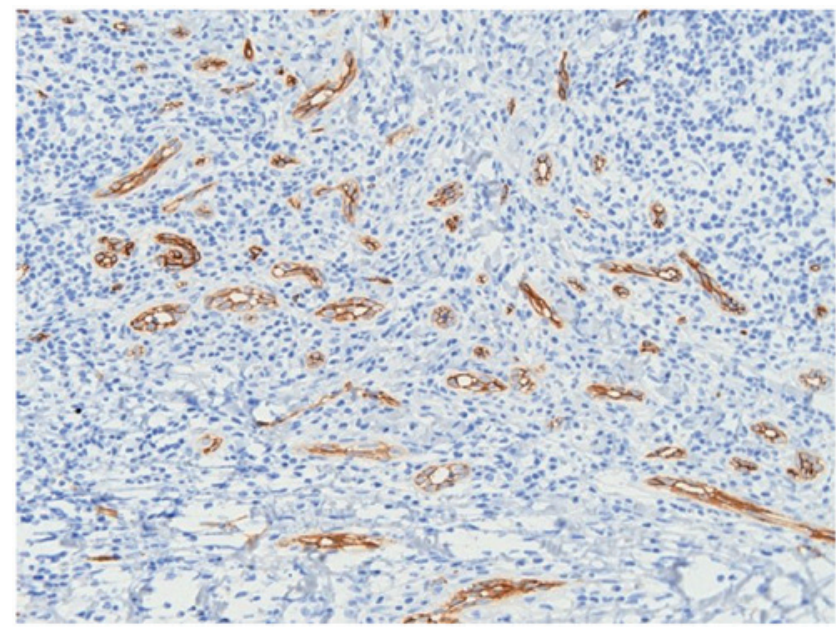

Figure 3. CD34 was positive in proliferative blood vessels (immunohistochemistry; magnification, $\mathrm{x} 200$ ).

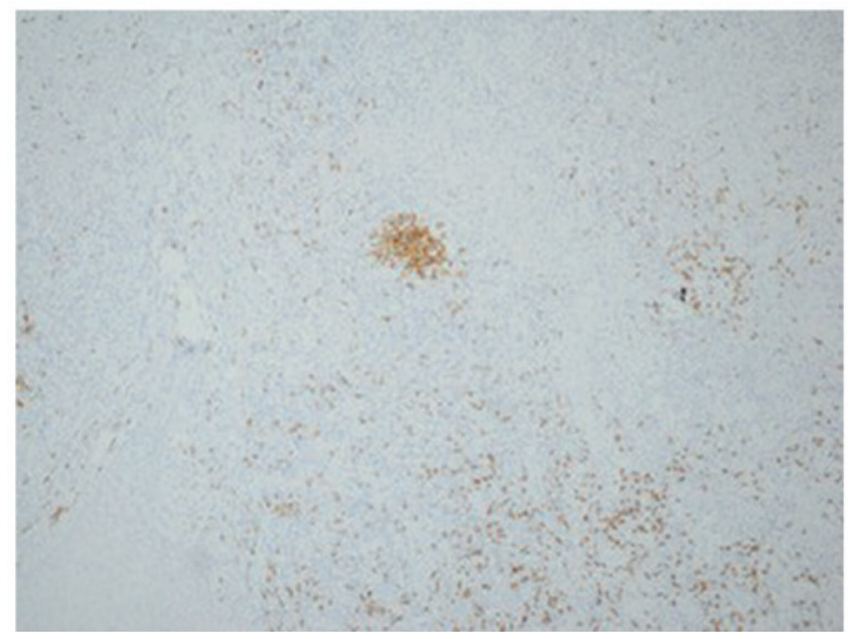

Figure 4. Lymphocytes surrounded the CD20-positiveblood vessels (immunohistochemistry; magnification, x100).

include pink-brown papules or nodules, single or multiple and $2-3 \mathrm{~cm}$ in diameter. AHLE may also occur in the mouth, trunk and extremities, and the vulva $(1,2)$; rare sites of involvement include the hands, breast, shoulder and oral mucosa (11). However, prior case reports have described incidents of ALHE affecting the penis (11) and the conjunctiva (12).

A diagnosis of ALHE was determined based on the clinical characteristics at manifestation and the histopathological features. The differential diagnosis included Kimura's disease, pyogenic granuloma (PG), Kaposi's sarcoma, lymphocytoma cutis, squamous cell carcinoma and angiosarcoma, among others. With the exception of Kimura's disease, other diseases with these pathological features are easy to distinguish from ALHE. Pathologically, both ALHE and Kimura's disease exhibit lymphocytic and acidophilic cell infiltration. However, there are important differences in the histopathology of ALHE and Kimura's disease. ALHE lesions typically present in the dermis and have histocyte-like or epithelioid vascular endothelial cells, an uneven thickness of the blood vessels and branching vascular hyperplasia. Conversely, the pathological changes in Kimura's 
disease occur primarily in the subcutaneous tissue, and have a characteristic lymphoid follicular structure $(13,14)$.

Herein is reported a case of ALHE, which occurs in the back only rarely. On visual inspection, the patient's lesion resembled a PG, whereas on palpation the texture was soft. Lymphoid follicles, eosinophils and branching vascular hyperplasia were not observed in the surrounding environment. A definitive diagnosis was reached following pathological examination. In the event a patient is admitted with an isolated convex neoplasm on the back, with features of hyperplasia scar tissue, occasional bleeding and acidophilic cells in the peripheral blood, the differential diagnosis must consider the possibility of ALHE.

ALHE has a generally favorable prognosis with a low associated mortality rate. The recommended treatment is surgical resection, and $\sim 33 \%$ of patients experience post-surgical recurrence (15). This type of disease is sensitive to deep tissue X-ray irradiation and electron beam therapy, but these methods carry the risk of inducing a malignant transformation. For patients unable to receive surgery or radiotherapy, glucocorticoid treatments are indicated. No cases of relapse were observed in patients during the follow-up period, following the complete surgical removal of the lesion.

\section{Acknowledgements}

The present study was supported by the Science and Technology Planning Project of Shenzhen, China (grant no. JCYJ20150402165325173).

\section{References}

1. Rapini RP, Bolognia JL and Jorizzo JL: Dermatology: 2-Volume. 2nd edition. Mosby Elsevier, St. Louis, 2007.

2. James WD, Elston D and Berger T: Andrew's diseases of the skin: Clinical Dermatology. Elsevier Health Sci, 2011.

3. Chitrapu P, Patel M, Readinger A and Menter A: Angiolymphoid hyperplasia with eosinophilia. Proc (Bayl Univ Med Cent) 27: 336-337, 2014.

4. Wells GC and Whimster IW: Subcutaneous angiolymphoid hyperplasia with eosinophilia. Br J Dermatol 81: 1-14, 1969.

5. Kaur T, Sandhu K, Gupta S, Kanwar AJ and Kumar B: Treatment of angiolymphoid hyperplasia with eosinophilia with the carbon dioxide laser. J Dermatolog Treat 15: 328-330, 2004.

6. Miller CJ, Ioffreda MD and Ammirati CT: Mohs micrographic surgery for angiolymphoid hyperplasia with eosinophilia. Dermatol Surg 30: 1169-1173, 2004.
7. Hollo P, Marschalko M, Sikos G, Harsing J and Horvath A: Angiolymphoid hyperplasia with eosinophilia in pregnancy. J Eur Acad Dermatol Venereol 19: 645-646, 2005.

8. Kempf W, Haeffner AC, Zepter K, Sander CA, Flaig MJ, Mueller B, Panizzon RG, Hardmeier T, Adams V and Burg G: Angiolymphoid hyperplasia with eosinophilia: Evidence for a T-cell lymphoproliferative origin. Hum Pathol 33: 1023-1029, 2002.

9. Shankar S and Russell-Jones R: Co-existence of lichen amyloidosus and angiolymphoid hyperplasia with eosinophilia. Clin Exp Dermatol 29: 363-365, 2004

10. Rajendran R, Padmakumar SK, Kothawar S and Nair B: Angiolymphoid hyperplasia with eosinophilia (ALHE). J Oral Maxillofac Pathol 9: 24-26, 2005.

11. Dewan P, Francis ND, Lear JT and Bunker CB: Angiolymphoid hyperplasia with eosinophilia affecting the penis. Br J Dermatol 159: 755-757, 2008.

12. Huang M, Lloyd WC III and O'Hara M: Angiolymphoid hyperplasia with eosinophilia: An unusual presentation in a child. J AAPOS 12: 302-304, 2008.

13. Filo V, Ferák I and Borecká D: Multiple unilateral reddish tumors on the ear and forehead in a woman with early syphilis. Angiolymphoid hyperplasia (ALHE) with eosinophilia coexisting with early latent syphilis. Arch Dermatol 130: 371-374, 1994.

14. Moy RL, Luftman DB, Nguyen QH and Amenta JS: Estrogen receptors and the response to sex hormones in angiolymphoid hyperplasia with eosinophilia. Arch Dermatol 128: 825-828, 1992.

15. Mukherjee B, Kadaskar J, Priyadarshini O, Krishnakumar S and Biswas J: Angiolymphoid hyperplasia with eosinophilia of the orbit and adnexa. Ocul Oncol Pathol 2: 40-47, 2015. 